\title{
Nanoscale
}

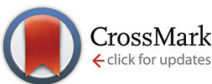

Cite this: Nanoscale, 2016, 8, 18032

Received 8th July 2016,

Accepted 6th October 2016

DOI: 10.1039/c6nr05419f

www.rsc.org/nanoscale

\section{Robust, directed assembly of fluorescent nanodiamonds $\uparrow$}

\author{
Mehran Kianinia, ${ }^{a}$ Olga Shimoni, ${ }^{a}$ Avi Bendavid, ${ }^{\mathrm{b}}$ Andreas W. Schell, ${ }^{\mathrm{c}}$ \\ Steven J. Randolph, ${ }^{d}$ Milos Toth, ${ }^{{ }^{a}}$ Igor Aharonovich ${ }^{{ }^{a}}$ and Charlene J. Lobo ${ }^{{ }^{a}}$
}

\begin{abstract}
Arrays of fluorescent nanoparticles are highly sought after for applications in sensing, nanophotonics and quantum communications. Here we present a simple and robust method of assembling fluorescent nanodiamonds into macroscopic arrays. Remarkably, the yield of this directed assembly process is greater than $90 \%$ and the assembled patterns withstand ultra-sonication for more than three hours. The assembly process is based on covalent bonding of carboxyl to amine functional carbon seeds and is applicable to any material, and to non-planar surfaces. Our results pave the way to directed assembly of sensors and nanophotonics devices.
\end{abstract}

Assembling fluorescent nanoparticles into macroscopic arrays is required for many applications spanning sensing, ${ }^{1,2}$ photonics, plasmonics and quantum information processing. ${ }^{3-5}$ To achieve this goal several top down techniques, including lithography $^{6,7}$ or dip-pen techniques, ${ }^{8,9}$ as well as bottom up methods using patterned self-assembled monolayers, ${ }^{10}$ electrostatic self-assembly ${ }^{11,12}$ and DNA origami ${ }^{13,14}$ have been developed. While these methods are capable of high resolution patterning of nanoparticle arrays, the assembled components are only weakly bonded to the substrate and cannot undergo further wet chemistry processing steps (e.g. sonication) or subsequent lithography. Such processing is often required for device applications where the fluorescent nanoparticles act as active components in microfluidic devices, ${ }^{15,16}$ as sensing probes ${ }^{17}$ or photon sources in which they are coupled to plasmonic structures or other optical elements. ${ }^{18-20}$

\footnotetext{
${ }^{a}$ School of Mathematical and Physical Sciences, University of Technology Sydney, P.O. Box 123, Broadway, NSW 2007 Australia.

E-mail: Charlene Lobo, charlene.lobo@uts.edu.au, igor.aharonovich@uts.edu.au, m.toth@uts.edu.au

${ }^{b}$ CSIRO Manufacturing, Lindfield, NSW 2070, Australia

${ }^{c}$ Department of Electronic Science and Engineering, Kyoto University, Kyoto daigaku-katsura, Nishikyo-ku, Kyoto, Japan

${ }^{d}$ FEI Company, 5350 NE Dawson Creek Dr., Hillsboro, OR 97124, USA

$\dagger$ Electronic supplementary information (ESI) available. See DOI: 10.1039/ c6nr05419f
}

In particular, there is a great interest in controlling and positioning fluorescent nanodiamonds that host nitrogen vacancy $\left(\mathrm{NV}^{-}\right)$defects, which can then be employed as nanoscale sensors for detection and imaging of weak magnetic fields, ${ }^{1,21,22}$ thermal imaging or thermometry ${ }^{23-25}$ and quantum measurements. ${ }^{26-28}$ Moreover, there is a great interest in assembling arrays of nanodiamonds ${ }^{29,30}$ that can subsequently be used to couple to plasmonic waveguides to realize quantum plasmonics circuitry. ${ }^{20,31,32}$ However, unlike gold nanoparticles, which can be assembled into arrays using SAMs and block copolymer templates, ${ }^{33}$ to date there is no robust method of accurately positioning nanodiamonds in arrays that can be subjected to further processing steps necessary for device fabrication.

Here we realize a facile, robust method for high resolution self-assembly of nanodiamonds which enables their use in sensing, photonic and quantum devices. We employ nanoscale seeds that are fabricated in a single step by a mask-free electron beam induced deposition (EBID) technique, ${ }^{34}$ terminate the seeds with amine groups, and self-assemble nanodiamonds into arrays defined by the seed positions. The technique is not limited to any specific substrate and can be used to position nanodiamonds on arbitrary materials and nonplanar surfaces. Finally, the technique offers high stability, which we demonstrate by subjecting the fabricated nanodiamond arrays to multiple sonication steps of up to 12 hours total duration.

The nanodiamond patterning process is illustrated in Fig. 1a and described in detail in ESI S1. $\dagger$ In step 1, electron beam induced deposition (EBID) was used to fabricate nanoscale carbonaceous seeds using the organic precursor naphthalene $\left(\mathrm{C}_{10} \mathrm{H}_{8}\right)$. EBID experiments were conducted using a differentially dry-pumped, field emission gun environmental SEM via a procedure described in ref. 35. Carbon seeds were deposited in arrays on a silicon substrate using a stationary defocused electron beam (15 keV, $300 \mathrm{pA}, 30 \mathrm{~s})$, resulting in disks of approximately $90 \mathrm{~nm}$ diameter and $20 \mathrm{~nm}$ height, as seen in Fig. $1 \mathrm{~b}$ and c. In step 2, the EBID seeds were aminefunctionalized by $45 \mathrm{~s}$ exposure to an ammonia plasma 

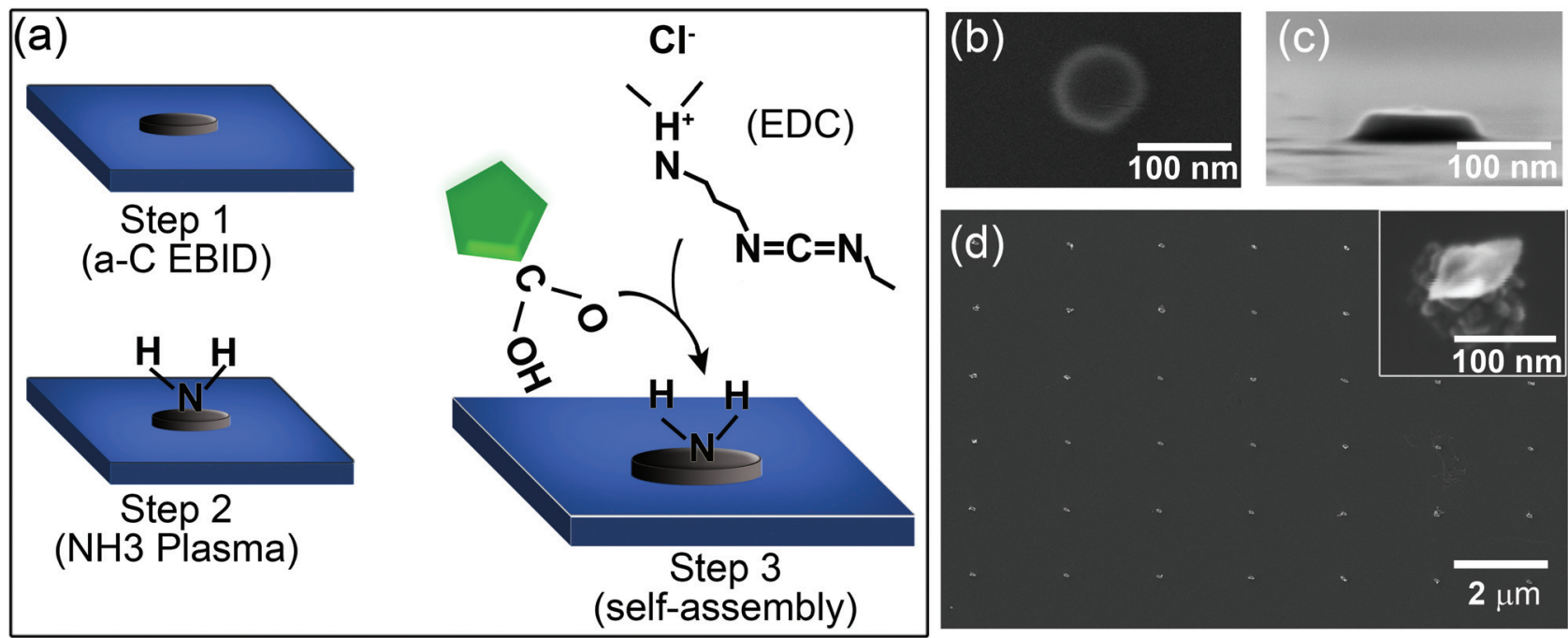

Fig. 1 Self-assembly of nanodiamonds on electron beam deposited carbon seeds. (a) Schematic of the process. (b, c) Plan and side view SEM images of amorphous carbon disks deposited by EBID. (d) SEM image of patterned area after nanodiamond attachment. The inset in (d) is a high magnification SEM image of an EBID seed with attached nanodiamonds.
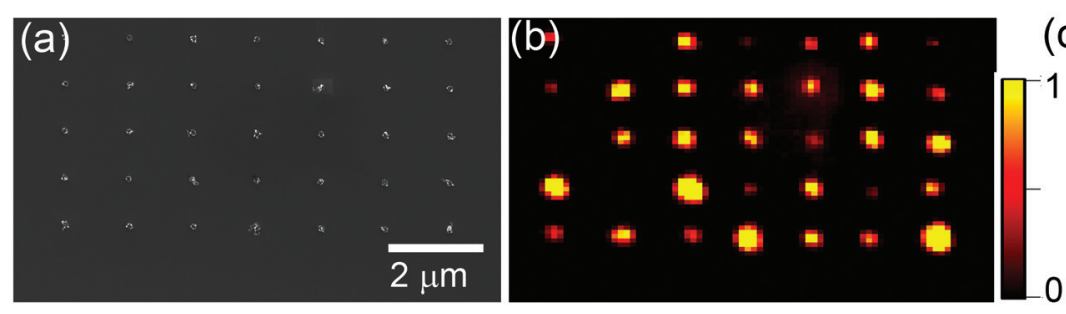

(c) 20

(d)
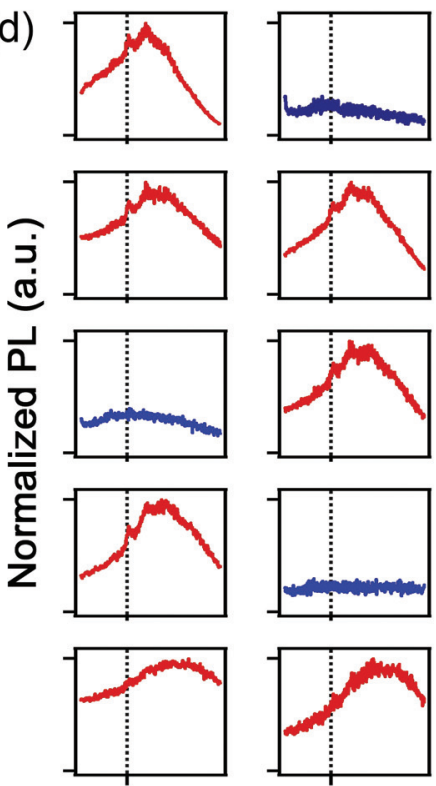
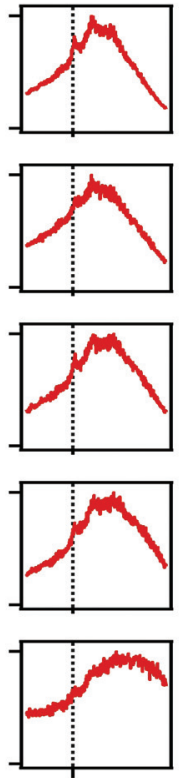

Wavelength $(\mathrm{nm})$
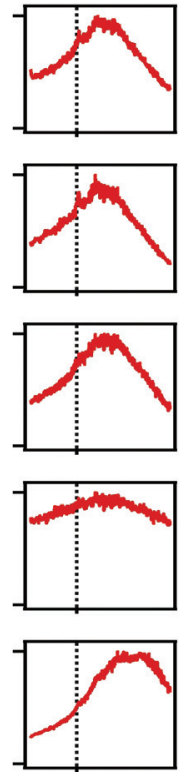
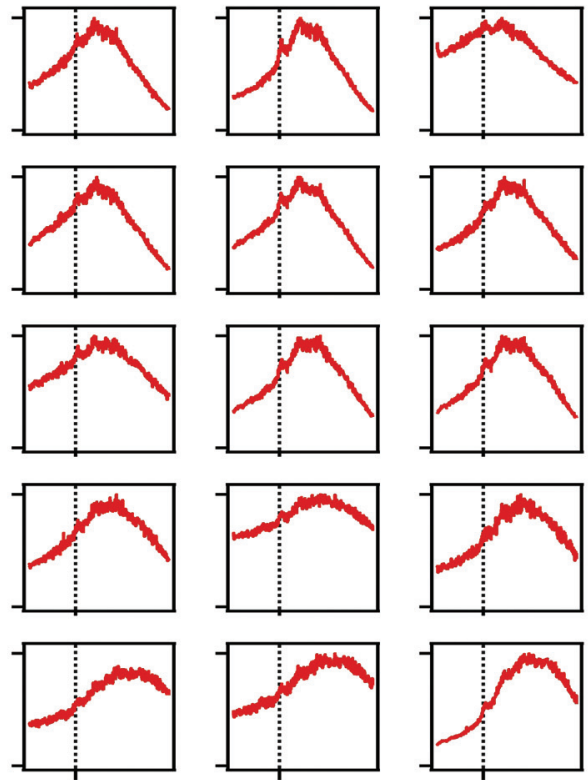

Fig. 2 Characterization of attachment yield and selectivity. (a) SEM image of a nanodiamond pattern. (b) Colour-coded confocal photoluminescence map of the same nanodiamond pattern. (c) Histogram of the PL intensities from each spot in the confocal map. (d) Corresponding normalized PL spectra from each individual spot showing the $\mathrm{NV}^{-}$emission at $637 \mathrm{~nm}$. The $\mathrm{NV}^{-}$zero phonon line is marked with a vertical dashed line for clarity. The adjacent broad emission is the phonon sideband. Only three locations in the patterned array do not show the NV ${ }^{-}$ZPL (blue $^{2}$ curves). 
generated in a Reactive Ion Etching (RIE) system operating at $100 \mathrm{~W}$ and $6 \mathrm{~Pa} \mathrm{NH}_{3}$. These conditions have been reported to produce the highest concentration of $\mathrm{NH}_{2}$ groups in the plasma. ${ }^{36}$ The extent and nature of amine groups created in the surface carbon was assessed by X-ray photoelectron spectroscopy (ESI S2 $\dagger$ ). The final step involves covalent attachment of $35 \mathrm{~nm}$ nanodiamonds to the EBID seeds using 1-ethyl-3-(3dimethylaminopropyl)carbodiimide (EDC). The presence of dangling bonds at the nanodiamond surface allows them to be functionalized with a variety of ligands. ${ }^{37,38}$ The preparation of the nanodiamonds used in the present study is described in detail elsewhere. ${ }^{39,40}$ The surfaces of these nanodiamonds are terminated with carboxylic acid $(-\mathrm{COOH})$ groups, enabling their attachment to amine-terminated surfaces through carbodiimide coupling chemistry. Conjugation was achieved by immersing the EBID-seeded Si substrate in an aqueous solution of EDC using the procedure detailed in ESI S1. $\dagger$ Fig. 1d and $\mathrm{S} 1 \dagger$ show the resulting patterned array of nanodiamonds on silicon and $\mathrm{Si}_{3} \mathrm{~N}_{4}$, respectively (the insets show high resolution images of single EBID seed with several nanodiamond crystals attached to each one).

To ascertain the nanodiamond attachment yield and selectivity of the technique, we employ confocal microscopy to obtain photoluminescence (PL) maps and spectra of the fabricated arrays. For the optical measurements, we employed a home built confocal microscope with a high numerical aperture objective $(100 \times, 0.9$ N.A), used for both excitation and collection of the emitted light. A $532 \mathrm{~nm}$ continuous wave laser was used for excitation, and all measurements were done at room temperature under ambient conditions. Fig. 2a shows a SEM image of a nanodiamond array and Fig. $2 \mathrm{~b}$ shows a con- focal map of the same array. The bright fluorescent spots correspond to the emission from nitrogen vacancy $\left(\mathrm{NV}^{-}\right)$ defects in the nanodiamonds. Variations in PL intensity seen in Fig. $2 \mathrm{~b}$ are caused primarily by the number of nanodiamonds attached to each carbon seed and variations in the number of NV centres within each nanodiamond. Fig. $2 \mathrm{c}$ is the histogram obtained from the confocal scan of Fig. $2 \mathrm{~b}$, representing the variation of fluorescence in a pattern produced with this method. Fig. 2d shows the spectrum recorded from each spot, demonstrating that 32 out of 35 locations have the characteristic emission from the $\mathrm{NV}^{-}$centres, equating to a $92 \%$ yield for the attachment process. Note that no nanodiamonds were attached in between the EBID seeds, giving the technique $100 \%$ selectivity.

We now study the effect of the nanodiamond concentration on the attachment yield. Fig. 3a shows a clear dependence of the yield on the initial concentration of fluorescent nanodiamonds. Increasing the nanodiamond concentration results in improved attachment yield. At a nanodiamond concentration of $2.5 \mu \mathrm{g} \mathrm{ml}{ }^{-1}$ the attachment yield was smaller than $5 \%$, increasing to $50 \%$ at a concentration of $12.5 \mu \mathrm{g} \mathrm{ml} \mathrm{m}^{-1}$. The optimum concentration was found to be $25 \mu \mathrm{g} \mathrm{ml}{ }^{-1}$, resulting in greater than $92 \%$ attachment, with only a few spots having no nanodiamonds. A higher concentration of nanodiamonds resulted in agglomeration with no increase in yield. The probabilities were deduced by analysis of confocal maps recorded under the same conditions as in Fig. 2 (and shown for each data point in Fig. 3). For all experiments, the ratio of EDC : nanodiamonds in the solution was fixed at $10: 1$.

The effectiveness of our method relies on covalent bonding between the amine and carboxyl functional groups on amor-
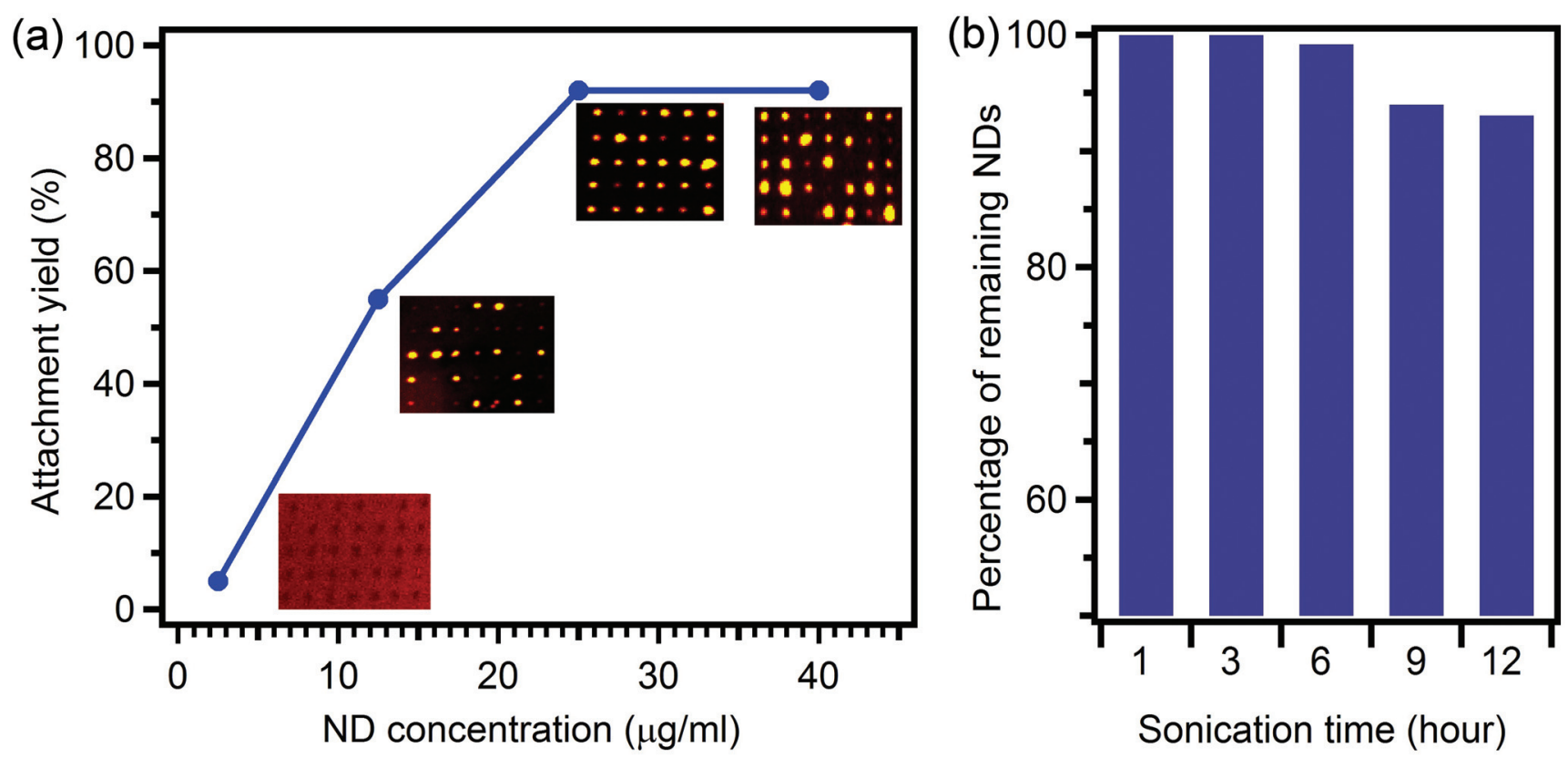

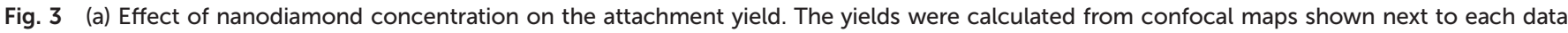
point. (b) Robustness of nanodiamond array under sonication. 
phous carbon and nanodiamond surfaces in the presence of EDC. We therefore expected the attached nanodiamonds to withstand further processing and treatment, as is required for many device applications. To examine the robustness of the technique, the assembled nanodiamonds were sonicated in a powerful ultrasonic bath (Bransonic 185 Watt Ultrasonic cleaner 221). Fig. 3b shows the remaining nanodiamonds after multi-step ultra-sonication for up to 12 hours. After three hours of sonication, all the nanodiamonds that were initially assembled were still attached to the substrate. Even after 12 hours, more than $90 \%$ of the self-assembled nanodiamonds remained on the surface, proving the unprecedented robustness of the assembly technique.

Finally, we demonstrate that the formed array can be used as a high-resolution magnetic field sensor, where each element in the array can serve as an individual pixel. Here, a microwave is guided through a $30 \mu \mathrm{m}$ wire and the $\mathrm{NV}^{-}$spin states are read out optically (so-called optically detected magnetic resonance, ODMR, described in ESI S3†) (Fig. 4a). ${ }^{41}$ Such sensing of magnetic fields in ambient environments is one of the most prominent applications of the $\mathrm{NV}^{-}$centre. Fig. $4 \mathrm{~b}$ shows a confocal map of the array of $\mathrm{NV}^{-}$centers. Fig. 4(c-e) shows three examples of optically detected magnetic resonance (ODMR) from randomly selected pixels in the array (marked with green circles). The red curves are nearly identical for all pixels and correspond to zero magnetic field. The green and the blue curves show the ODMR under $1 \mathrm{mT}$ and $3 \mathrm{mT}$ magnetic field. Each pixel shows a distinct ODMR signal that can then be used to deduce the local magnetic field in the proximity of the pixel. Note that since the measurement is done with an ensemble of nanodiamonds, the signal is broadened, with each dip comprising several lines from different $\mathrm{NV}^{-}$ centers at each spot in the array. This technique is ideal to test for local absorption of metal nanoparticles or the presence of foreign para- and ferromagnetic metals. In principle, our technique can also be applied to nanodiamonds with single emitters, which are advantageous for quantum photonic applications. (a)

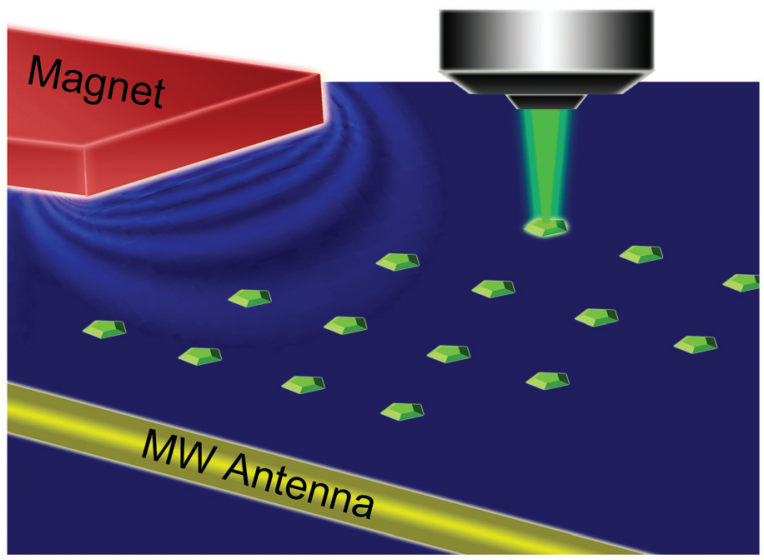

(b)

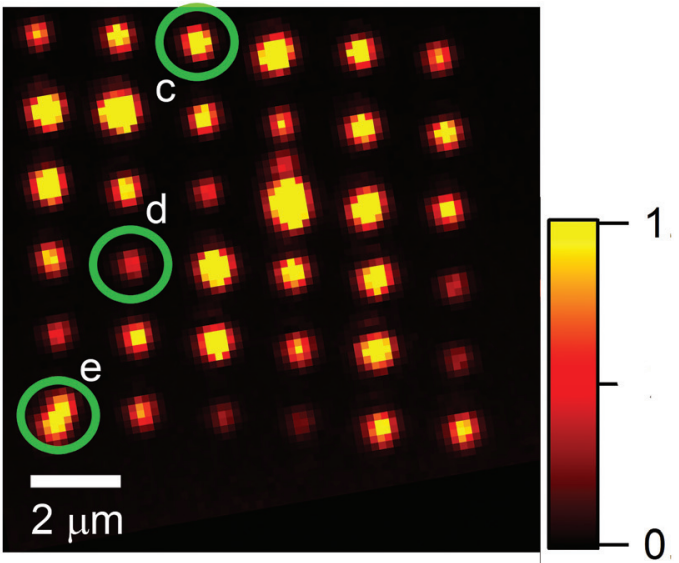

(c)

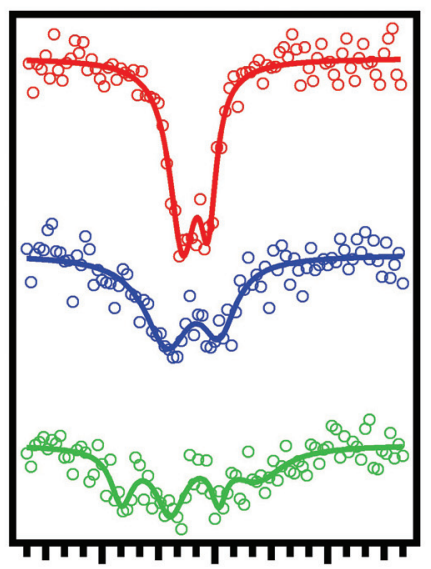

$\begin{array}{lll}2.82 & 2.88 & 2.94\end{array}$ MW Frequency $(\mathrm{GHz})$ (d)

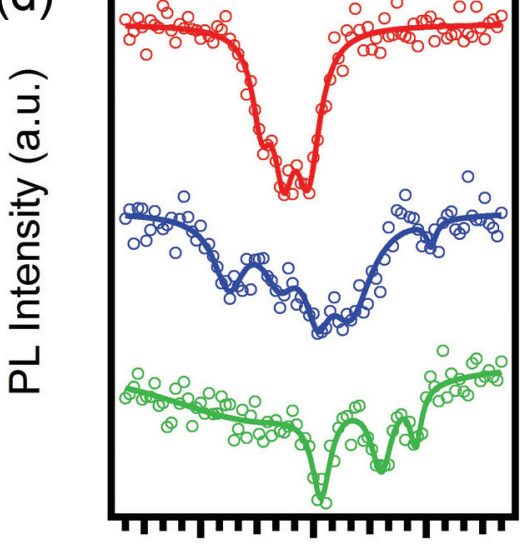

$2.82 \quad 2.88 \quad 2.94$ MW Frequency $(\mathrm{GHz})$

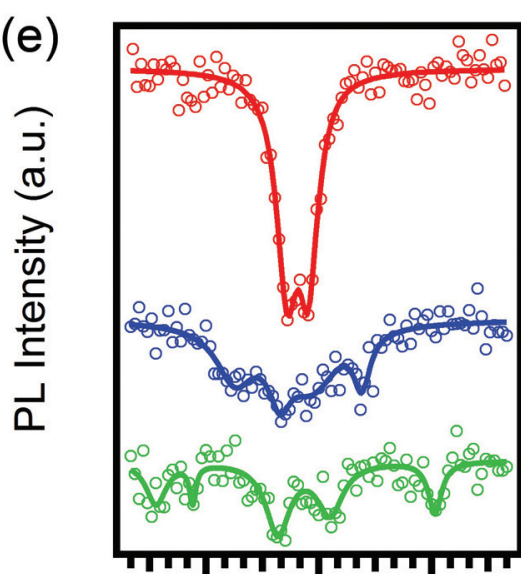

$\begin{array}{lll}2.82 & 2.88 & 2.94\end{array}$ MW Frequency $(\mathrm{GHz})$

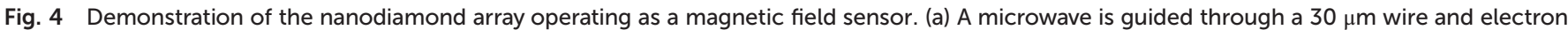

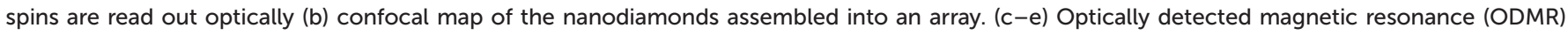

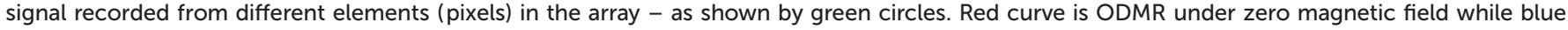
and green curves correspond to 1 and $3 \mathrm{mT}$ fields, respectively. 
In conclusion we have developed a facile, generic technique for directed assembly of fluorescent nanodiamonds into robust arrays. The assembly technique has greater than 90 percent efficiency. Moreover, the nanodiamonds are covalently bonded and stay in their positions even after repeated ultrasonication treatments, making the technique very attractive for practical device applications. Finally, we have performed a proof of principle sensing measurement of various magnetic fields to show that each pixel in the array can be used as an independent magnetic field sensor. Larger nanoparticle arrays, as well as serial device production can, in principle, be achieved using a multibeam scanning electron microscope and thin passivation layers applied between sequential processing steps. Our method paves the way to realization of scalable platforms for sensing or integrated quantum photonics, where there is a real need for robust, large area assembly of fluorescent nanoparticles.

\section{Author contributions}

C. J. L. and S. J. R. conceived the idea, C. J. L., I. A., O. S., M. K., S. J. R. and M. T. designed the experiments, M. K. and A. B. conducted the experiments, A. W. S. contributed towards the ODMR measurements and C. J. L., I. A. and M. K. wrote the manuscript. All authors have discussed the results and given approval to the final version of the manuscript.

\section{Acknowledgements}

This work was funded by FEI Company and the Australian Research Council. The authors thank Hugh Mackay for assistance with EBID seed fabrication.

\section{References}

1 S. Steinert, F. Dolde, P. Neumann, A. Aird, B. Naydenov, G. Balasubramanian, F. Jelezko and J. Wrachtrup, Rev. Sci. Instrum., 2010, 81(4), 043705.

2 Y. Wu, F. Jelezko, M. B. Plenio and T. Weil, Angew. Chem., Int. Ed., 2016, 55(23), 6586-6598.

3 C. Santori, P. E. Barclay, K. M. Fu, R. G. Beausoleil, S. Spillane and M. Fisch, Nanotechnology, 2010, 21(27), 274008.

4 G. D. Fuchs, G. Burkard, P. V. Klimov and D. D. Awschalom, Nat. Phys., 2011, 7(10), 789-793.

5 L. Childress and R. Hanson, MRS Bull., 2013, 38(02), 134138.

6 V. R. Manfrinato, D. D. Wanger, D. B. Strasfeld, H. S. Han, F. Marsili, J. P. Arrieta, T. S. Mentzel, M. G. Bawendi and K. K. Berggren, Nanotechnology, 2013, 24(12), 125302.

7 W. Xie, R. Gomes, T. Aubert, S. Bisschop, Y. Zhu, Z. Hens, E. Brainis and D. Van Thourhout, Nano Lett., 2015, 15(11), 7481-7487.

8 Y. Wang, Y. Zhang, B. Li, J. Lü and J. Hu, Appl. Phys. Lett., 2007, 90(13), 133102.
9 P. Maletinsky, S. Hong, M. S. Grinolds, B. Hausmann, M. D. Lukin, R. L. Walsworth, M. Loncar and A. Yacoby, Nat. Nanotechnol., 2012, 7(5), 320-324.

10 S. G. Rao, A. Karim, J. Schwartz, N. Antler, T. Schenkel and I. Siddiqi, ACS Appl. Mater. Interfaces, 2014, 6(15), 1289312900.

11 M. Jiang, J. A. Kurvits, Y. Lu, A. V. Nurmikko and R. Zia, Nano Lett., 2015, 15(8), 5010-5016.

12 M. Kolibal, M. Konecny, F. Ligmajer, D. Skoda, T. Vystavel, J. Zlamal, P. Varga and T. Sikola, ACS Nano, 2012, 6(11), 10098-10106.

13 A. Gopinath, E. Miyazono, A. Faraon and P. W. Rothemund, Nature, 2016, 535(7612), 401-405.

14 T. Zhang, A. Neumann, J. Lindlau, Y. Wu, G. Pramanik, B. Naydenov, F. Jelezko, F. Schuder, S. Huber, M. Huber, F. Stehr, A. Hogele, T. Weil and T. Liedl, J. Am. Chem. Soc., 2015, 137(31), 9776-9779.

15 P. Andrich, B. J. Aleman, J. C. Lee, K. Ohno, C. F. de las Casas, F. J. Heremans, E. L. Hu and D. D. Awschalom, Nano Lett., 2014, 14(9), 4959-4964.

16 S. Steinert, F. Ziem, L. T. Hall, A. Zappe, M. Schweikert, N. Gotz, A. Aird, G. Balasubramanian, L. Hollenberg and J. Wrachtrup, Nat. Commun., 2013, 4, 1607.

17 H. J. Mamin, M. Kim, M. H. Sherwood, C. T. Rettner, K. Ohno, D. D. Awschalom and D. Rugar, Science, 2013, 339(6119), 557-560.

18 T. Schroeder, A. W. Schell, G. Kewes, T. Aichele and O. Benson, Nano Lett., 2011, 11(1), 198-202.

19 A. Huck, S. Kumar, A. Shakoor and U. L. Anderson, Phys. Rev. Lett., 2011, 106(9), 096801-096801.

20 T. T. Tran, J. Fang, H. Zhang, P. Rath, K. Bray, R. G. Sandstrom, O. Shimoni, M. Toth and I. Aharonovich, Adv. Mater., 2015, 27(27), 4048-4053.

21 J. M. Taylor, P. Cappellaro, L. Childress, L. Jiang, D. Budker, P. R. Hemmer, A. Yacoby, R. Walsworth and M. D. Lukin, Nat. Phys., 2008, 4(10), 810-816.

22 S. Hong, M. S. Grinolds, L. M. Pham, D. Le Sage, L. Luan, R. L. Walsworth and A. Yacoby, MRS Bull., 2013, 38(02), 155-161.

23 J. P. Tetienne, A. Lombard, D. A. Simpson, C. Ritchie, J. Lu, P. Mulvaney and L. C. Hollenberg, Nano Lett., 2016, 16(1), 326-333.

24 G. Kucsko, P. C. Maurer, N. Y. Yao, M. Kubo, H. J. Noh, P. K. Lo, H. Park and M. D. Lukin, Nature, 2013, 500(7460), $54-58$.

25 P. Neumann, I. Jakobi, F. Dolde, C. Burk, R. Reuter, G. Waldherr, J. Honert, T. Wolf, A. Brunner and J. H. Shim, Nano Lett., 2013, 13(6), 2738-2742.

26 L. P. McGuinness, Y. Yan, A. Stacey, D. A. Simpson, L. T. Hall, D. Maclaurin, S. Prawer, P. Mulvaney, J. Wrachtrup, F. Caruso, R. E. Scholten and L. C. Hollenberg, Nat. Nanotechnol., 2011, 6(6), 358-363.

27 A. W. Schell, G. Kewes, T. Hanke, A. Leitenstorfer, R. Bratschitsch, O. Benson and T. Aichele, Opt. Express, 2011, 19(8), 7914-7920.

28 O. Benson, Nature, 2011, 480(7376), 193-199. 
29 O. Shimoni, J. Cervenka, T. J. Karle, K. Fox, B. C. Gibson, S. Tomljenovic-Hanic, A. D. Greentree and S. Prawer, ACS Appl. Mater. Interfaces, 2014, 6(11), 8894-8902.

30 I. Aharonovich, J. C. Lee, A. P. Magyar, D. O. Bracher and E. L. Hu, Laser Photonics Rev., 2013, 7(5), L61-L65.

31 R. W. Heeres, L. P. Kouwenhoven and V. Zwiller, Nat. Nanotechnol., 2013, 8(10), 719-722.

32 M.-C. Dheur, E. Devaux, T. W. Ebbesen, A. Baron, J.-C. Rodier, J.-P. Hugonin, P. Lalanne, J.-J. Greffet, G. Messin and F. Marquier, Sci. Adv., 2016, 2, e1501574.

33 F. Liu, E. J. Luber, L. A. Huck, B. C. Olsen and J. M. Buriak, ACS Nano, 2015, 9, 2184-2193.

34 I. Utke, P. Hoffmann and J. Melngailis, J. Vac. Sci. Technol., B: Microelectron. Nanometer Struct. - Process., Meas., Phenom., 2008, 26(4), 1197.
35 C. J. Lobo, A. Martin, M. R. Phillips and M. Toth, Nanotechnology, 2012, 23(37), 375302.

36 M. L. Steen, K. R. Kull and E. R. Fisher, J. Appl. Phys., 2002, 92(1), 55.

37 Y. Liang, M. Ozawa and A. Krueger, ACS Nano, 2009, 3(8), 2288-2296.

38 A. Krüger, Y. Liang, G. Jarre and J. Stegk, J. Mater. Chem., 2006, 16(24), 2322-2328.

39 S.-J. Yu, M.-W. Kang, H.-C. Chang, K.-M. Chen and Y.-C. Yu, J. Am. Chem. Soc., 2005, 127(50), 17604-17605.

40 M. Montalti, A. Cantelli and G. Battistelli, Chem. Soc. Rev., 2015, 44(14), 4853-4921.

41 A. Gruber, A. Dräbenstedt, C. Tietz, L. Fleury, J. Wrachtrup and C. V. Borczyskowski, Science, 1997, 276(5321), 20122014. 